Infusionsther Transfusionsmed 1995;22:137-138

\title{
Impressum, Vol. 22, No. 3, 1995
}

Infusionstherapie und Transfusionsmedizin

Gründungsherausgeber

H. Reissigl, Innsbruck

Herausgeber

J. Eckart, Augsburg V. Kretschmer, Marburg W. Mempel, München K. Meßmer, München K.

Peter, München W. Stangel, Hannover K.-H. Usadel, Frankfurt

Schriftleiter

H. Forst, Augsburg B. Zwißler, München

Wissenschaftlicher Beirat

M. Adolph, Augsburg

FW. Ahnefeld, Ulm

J. Askanazi, New York

C. Baldamus, Köln

H. Bardenheuer, Heidelberg

J.-F Baron, Paris

W. Behrendt, Aachen

H. Borberg, Köln

U. B. Bruckner, Ulm

R. Eckstein, Erlangen

A. Encke, Frankfurt

U. Finsterer, München

L. Frey, München

Y. Fujita, Okayama

L. Gattinoni, Mailand

M. Georgieff, Ulm

A. Grünert, Ulm

H. J. Gurland, München

P. Hanfland, Bonn

M. Heberer, Basel

J. P. Isbister, Sydney

K. Jauch, München

W.-P. Kloevekorn, Bad Nauheim

H.-J. Kolb, München

W. Kox, Berlin

G. C. Kramer, Galveston

H. G. Kress, Wien

B. Kubanek, Ulm

P. Kühnl, Hamburg

H. Laubenthal, Bochum 
N. Mutz, Innsbruck

U E. Nydegger, Bern

W. Oettinger, Trier

Th. Prien, Münster

D. Roelcke, Heidelberg

R. Roos, München

W. Schleinzer, Hamburg

D. Schönitzer, Innsbruck

H.-G Sieberth, Aachen

U. Suchner, München

L. Sunder-Plassmann, Ulm

E. Wenzel, Homburg/Saar

K. Werdan, Halle/Saale

K. Widhalm, Wien

G. Wolfram, München R. Zander, Mainz

KARGEK

Bibliographischer Hinweis:

Inhaltsverzeichmsse dieser Zeitschrift erscheinen regelmäßig in current contents ${ }^{\circledR}$ sowie in anderen bibliographischen Diensten.

S. Karger $\square$ Medical and Scientific Publishers $\square$ Basel · Freiburg · Paris $\square$ London $\mathbf{n}$ New York · New Delhi $\square$ Bangkok $\square$ Singapore $\square$ Tokyo $\cdot$ Sydney

Die Zeitschrift erscheint zweiraonatlich; pro Jahr erscheint 1 Band zu je 6 Heften. DGTl-

Mitglieder erhalten die Zeitschrift irn Rahmen ihrer Mitgliedschaft. Bezugspreis für Jahrgang 22, 1995, DM 173-/SFr 135,-, einschließlich MwSt., zuzüglich Postgebühren. Der

Abonnementpieis ist im voraus zahlbar. Das Abonnement der Zeitschrift läuft weiter, wenn es nicht spätestens 4 Wochen vor Abschluß eines Bandes abbestellt wird. Abonnementbestellungen können bei jeder Buchhandlung oder direkt beim Verlag aufgegeben werden:

Bundesrepublik Deutschland: S. Karger GmbH Lörracher Str. 16 a D-79115 Freiburg Telefon (0761) 452070 Telefax (0761) 4520714 Postgiro München 61440-808

Übrige Lander: S. Karger AG Allschwilerstr. 10 Postfach CH-4009 Basel Telefon (061) 306111

Telefax (061) 3061234 E-Mail Karger@Karger.ch

Anzeigen: S. Karger

Verlag für Medizin und Naturwissenschaften $\mathrm{GmbH}$

Lörracher Str. 16a, D-79115 Freiburg

Telefon (0761) 452070

Gültig ist die Preisliste Nr. 11 vom 1. Januar 1994.

Für den Inhalt außerhalb des redaktionellen Teiles (insbesondere Anzeigen,

Industrieinformationen, Presse-zitate und Kongreßinformationen) übernehmen Schrift-leitung, Beirat und Verlag keine Gewähr. Eine Markenbezeichnung kann warenzeichenrechtlich geschützt sein, auch wenn bei ihrer Verwendung in dieser Zeitschrift das Zeichen ${ }^{\circledR}$ oder ein anderer Hinweis auf etwa bestehende Schutzrechte fehlen sollte. Für Satz-fehler, insbesondere bei Dosierungsangaben, wird keine Gewähr übernommen.

Die Zeitschrift sowie alle in ihr enthaltenen einzelnen Beiträge und Abbildungen sind urheberrechtlich geschützt. Jede Verwertung. die nicht ausdrücklich vom Urheberrechtsgesetz zugelassen ist, bedarf der vorherigen Zustimmung des Verlags. Das gilt insbesondere für Vervielfältigungen, Bearbeitungen, Übersetzungen, Mikro-verfilmungen und die Einspeicherung 
und Verarbeitung in elektronischen Systemen. Fotokopien dürfen nur für den persönlichen Gebrauch als Einzelkopien hergestellt werden. Jede im Bereich eines gewerblichen Unternehmens zulässig hergestellte oder benutzte Kopie dient gewerblichen Zwecken gem. § 54(2) UrhG und verpflich-tet zur Gebührenzahlung an die Verwertungsgesellschaft WORT Abt. VG Wissenschaft, Goethestraße 49, D-80336 München

(C) Copyright 1995 by S. Karger

Verlag für Medizin und Naturwissenschaften $\mathrm{GmbH}$

Lörracher Str. 16 a, D-79115 Freiburg

Verlagsleitung und Presserechtlich verantwortlich: Sibylle Hopf Herstellung: Georg Brunner Redaktionsassistenz: Martina Zeller Anzeigenverwaltung: Christiane Opitz

Satz und Druck: Walter Biering GmbH Grafischer Betrieb Freisinger Landstraße 21 D-80939 München 